\title{
Image Registration: A Review of Elastic Registration Methods Applied to Medical Imaging
}

\author{
Asmita A Moghe, PhD. \\ Associate Professor \\ Department of Information Technology \\ UIT RGPV Bhopal
}

\author{
Jyoti Singhai, $\mathrm{PhD}$ \\ Associate Professor \\ Department of Electronics \& Communication \\ MANIT Bhopal
}

\begin{abstract}
Image registration is mapping different images of the same scene which have undergone changes due to time, position or inherent changes in the image. In medical images these may be due to motion artifact, breathing, heartbeat, etc which are difficult to register only by rigid registration and need a local transformation to correct the deformations that are elastic in nature at the local level. This is corrected by applying elastic registration applied to the initially rigid registered images. Such mapping of coordinates of the image that has undergone transformation on account of one or a combination of these factors calls for hybrid registration. A number of elastic registration methods as applied to medical imaging have been discussed in this paper and results for hybrid registration of pre contrast and post contrast abdominal CT images are shown. The resulting images show increase in correlation coefficient and Mutual information after hybrid registration, a decrease in Mean square error with differences being minimised after registration. This helps in improving diagnostic accuracy.
\end{abstract}

\section{General Terms}

Image processing, Pattern recognition, Image matching

\section{Keywords}

Image registration, Elastic registration, Non rigid registration, Feature points, Mutual information, Thin plate splines.

\section{INTRODUCTION}

The objective of image registration is to bring different images of the same scene into same spatial coordinates. Images under consideration are images of the same scene usually called the reference and study images wherein the study image is compared to the reference image. Study image is the image that may have been acquired at a different point of time, from a different angle or may have been acquired from a different sensor or undergone some deformation on its own compared to that of the reference image. In such a situation matching or comparison of the two images to detect the change becomes difficult because the reference and study images are not in same spatial coordinates. Image registration is the process that helps to map the coordinates of the study image to that of the reference image [1].

Registration is necessary for (i) integrating information obtained from different sensors (ii) extracting changes in images taken at different time or under different conditions (iii) for 3 dimensional reconstruction of images taken from different views. Registration methods can broadly be categorized into three research areas. It is used in (i) Computer vision and Pattern recognition for the purpose of segmentation, object recognition, motion tracking, character recognition etc. (ii) Remote Sensing to obtain valuable information in fields like agriculture, oceanography, mineral exploration, forestry etc. and (iii) Medical Image Analysis for tumor detection, dose administration, localization of disease etc.

\subsection{Image registration in medical imaging}

Most of the work in medical image registration has seen tremendous growth since 1980's [2][3] with new and efficient techniques [4] still being explored. Josien P.W. Pluim and J.Michael Fitzpatrick [5] surveyed the publications on medical image registration from 1988 till 2003 using PubMed and ISI Web of Science. They found that publications on medical image registration greatly increased from 1993 to 2003, showing more publications using PET and SPECT images were available with publications in CT and MRI being relatively constant in that decade. IT also brought forth that intra operative and elastic registration had tremendous scope for research.

In medical imaging, registration has been used for combining information from different modalities for example to register cardiac images from different modalities [6] and in minimally invasive spine surgery [7], for navigational guidance in orthopedic surgery [8] etc. Image registration is also used for improving diagnostic accuracy for example in locating lung nodules in chest radiograph images by suppressing ribs and clavicles [9], to observe interval growth in the lung nodules in chest during initial and follow up CT (Computed Tomography) scan [10], compensate for artifacts due to patient movement during Digital subtraction angiography [11] for registering fixed and moving ultrasound images to correct deformation due to patient positioning, heartbeat, respiration to guide in intra operative surgical applications[12],[13], registering x-ray mammography images for detection of breast cancer in early stages, diagnosing benign and malignant tissues in breast using 3D MRI (Magnetic resonance imaging) [14] or even for retinal eye fundus images in [15] during diabetic retinotherapy to evaluate growth of retinal diseases.

\subsection{Rigid and Elastic registration}

In medical imaging, initially most of the work was confined to rigid registration of brain images because there is relatively little change in brain shape or position within the skull over the relatively short periods between scans. Only translation or rotation transformation on the image is able to correct the rotational or translational differences in the images. Rigid registration was then extended to affine registration to include shear and scaling differences in the images. Further non rigid 
registration was applied to images of more deformable organs [16] like liver, kidney, heart etc. In brain, the structure of skull, ribs in the chest, bones in orthopaedic images provide rigid landmarks or features for registration. Challenge in registration of images of involuntary organs is unavailability of such rigid features. These images are not only affected by global deformations but also suffer from local non rigid deformations. Hence to register medical images having involuntary organs like abdominal CT images, hybrid registration is needed where initial registration is needed to correct translation and rotational errors during acquisition and elastic registration to correct non linear deformations. Elastic registration is seen to be an area of work that is being actively explored.

\section{ELASTIC REGISTRATION APPLIED TO MEDICAL IMAGING}

In medical images, elastic transformation may be required in case of biological changes in the anatomy over the passage of time or due to injection of some fluid called contrast agent for assessing the anatomy, patient movement, during breathing etc. Such deformations are corrected using non rigid registration technique [3]. Non rigid registration technique consists of two stages Initial registration and elastic registration. Initial registration corrects global changes like translation and rotation which occurred during image acquisition. Elastic registration provides localized stretching of images to correct local non linear deformations.

Many efforts have gone in this direction starting from applying elastic registration for brain images like use of combination of intensity based registration at global level and affine registration at local level to correct local deformations [17], use of wavelet decomposition to reduce computational complexity registering MR images at local level my maximizing mutual information [18]. A variation to this was presented in [19] where initial registration was intensity based but local affine registration made use of features extracted which were the centres of sub-images registered initially. Pixel information of overlapping boundaries was however lost. This problem was corrected in [20] by use of a different sub division of quadrants. The methods also used Thin plate splines for elastically registering the MR images using the obtained feature points. Hybrid registration of monomodal thorax CT images [21] uses anatomical feature points located on spine etc. to detect the lung nodules. In [22] abdominal CT images were used for automatic hybrid registration with edge centroids and end points with maximum and minimum distance to edge centroids being used as feature points to register intra patient images. Use of $\mathrm{B}$ splines for elastic registration is made in [23] and [24]. In [24] 2D and 3D MR as well as CT brain images are registered using synthetically warped images. Intensity independent automatic registration of brain images is seen in [25] that uses cubic splines for elastic registration. Elastic registration is also applied to inter frame abdominal images for kidney reconstruction using image morphology in [26]. To choose the transformation function for application to elastic registration, a comparative study of transfer functions like weighted mean (WM), piecewise linear (PL), Thin plate splines (TPS), multiquadric (MQ) etc. can be observed in [27].

\section{METHODOLOGY USED}

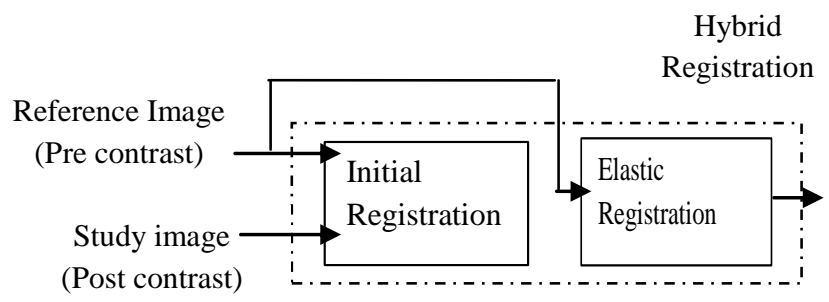

Figure 1: Block diagram of hybrid image registration

The method adopted uses intensity based initial registration to correct translation and rotational differences. This is achieved using wavelet decomposition as in (1) with decomposition being carried out up to 3 levels. In equation (1), the wavelet decomposition of a function $\mathrm{f}(\mathrm{x}) \in \mathrm{L}^{2}(\mathfrak{R})$ is defined [22] as follows.

$\left\langle W_{\psi} f(x)(s, \tau)\right\rangle=\left\langle f(x), \psi_{s \tau}(x)\right\rangle=\frac{1}{\sqrt{|s|}} \int_{\Re} f(x) \psi\left(\frac{x-\tau}{s}\right) d x(1)$

Here $\psi_{s \tau}(x)$ defines the family of wavelet functions with $(s, \tau) \in \mathfrak{R}$ Here $s \neq 0$ is the dilation parameter and $\tau$ is the translation parameter. Wavelets $\psi_{s \tau}(x)$ are obtained from mother wavelet $\psi(x)$ by dilation and translation [28] as given in equation (2).

$\psi_{s \tau}(x)=\sqrt{2^{-s}} \psi\left(2^{-s} x-\tau\right)$

$\mathrm{dB} 8$ wavelet has been used for decomposition. The images are then registered for given rotation keeping translation the same at each level till the original level. They are said to be registered when mutual information (MI) between them is maximised. Normalized mutual information [3] $I_{N}$ that is used is as given in equation (3) where $A$ and $B$ are the two random variables associated with pre contrast and post contrast images

$I_{N}(A, B)=\frac{H(A)+H(B)}{H(A, B)}$

$H(A)$ and $H(B)$ are the entropies of random variables $A$

and $B$ which give the amount of uncertainty associated with the random variable and $H(A, B)$ their joint entropy.

The initial registered image so obtained now needs correction at local level which requires segmentation to access the lesions in the image. So, initial registered post contrast image and the reference pre contrast image are segmented using thresholding as in [29]. Segmentation is followed by morphological opening, closing and fill operations to smooth the images. Then feature points like centroids of segmented lesions in the two images, image centroid and lesion hull points [30] are located in both the images. Corresponding feature points in the two images are then used for elastically registering the post contrast image to the pre contrast image using Thin plate splines [31] having interpolating function as given in equation (4).

$f(x, y)=a_{1}+a_{x} x+a_{y} y+\sum_{i=1}^{n} w_{i} U\left(\left|P_{i}-(x, y)\right|\right)$

Here coefficients $a_{1}, a_{x}, a_{y}$ represent the affine parameters;

coefficients $w_{i}$ represent the warped parameters due to elastic 
deformations in the image and $P_{i}$ are feature points in reference image. The final image so obtained after initial and elastic registration is the hybrid registered image.

Results of registration can be verified qualitatively by observing the difference in original pre and post contrast images, between pre contrast and initial registered image and between pre contrast and hybrid registered image. Superimposition of registered image (initial and hybrid) on unregistered image also shows the variations clearly in section 4.0. To evaluate the registration results quantitatively, Correlation coefficient, Mutual information and Mean square error (MSE) have been used as in section 4.0.

Correlation coefficient is computed according to equation (5). In equation (5), $\left(f(x, y)\right.$ and $\left(f^{\prime}(x, y)\right.$ are the pre contrast and post contrast images and $\overline{f(x, y)}$ and $\overline{f^{\prime}(x, y)}$ are their mean values respectively.

$$
C C=\frac{\sum_{m} \sum_{n}(f(x, y)-\overline{f(x, y)})\left(f^{\prime}(x, y)-\overline{f^{\prime}(x, y)}\right)}{\left.\sqrt{\left(\sum_{m} \sum_{n}(f(x, y)-\overline{f(x, y)})^{2}\right)\left(\sum_{m} \sum_{n}\left(f^{\prime}(x, y)-\overline{f^{\prime}(x, y)}\right)^{2}\right.}\right)}(5)
$$

The mean square error is computed as in equation (6)

$$
M S E=\frac{1}{M N} \sum_{y=1}^{M} \sum_{x=1}^{N}\left[f(x, y)-f^{\prime}(x, y)\right]^{2}
$$

where images $f$ and $f^{\prime}$ are of size $M \times N . f(x, y)$ is the reference image and $f^{\prime}(x, y)$ the study image.

\section{EXPERIMENTAL RESULTS}

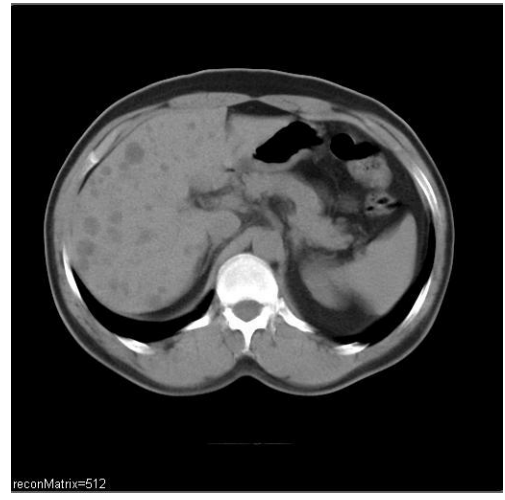

a

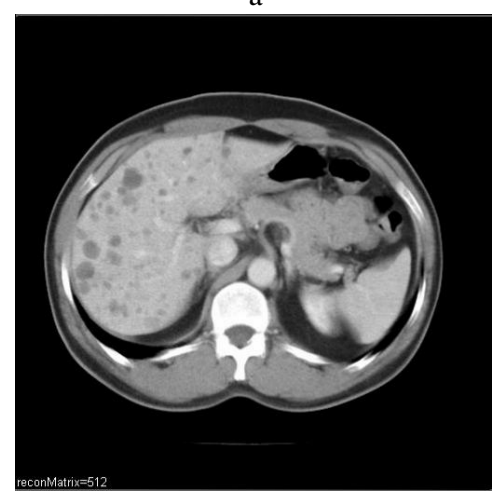

d

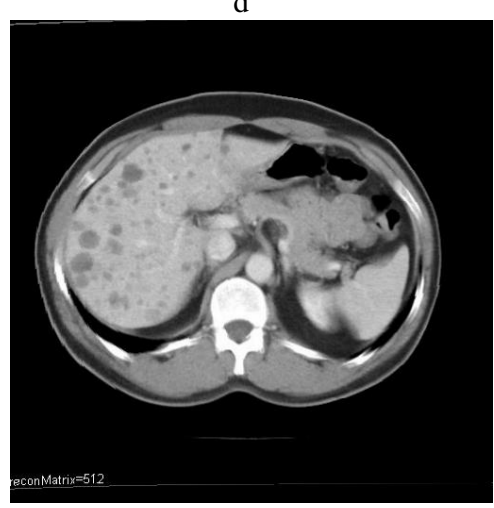

g

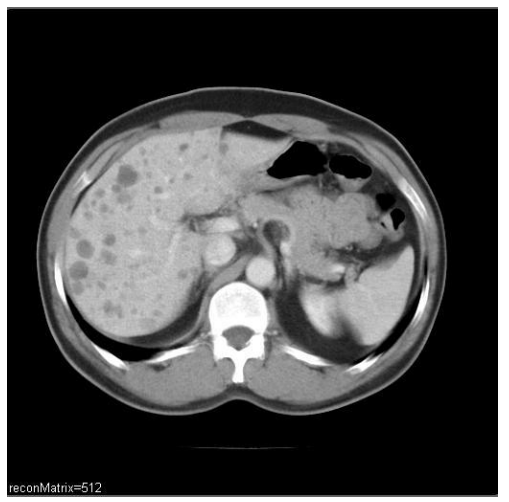

b

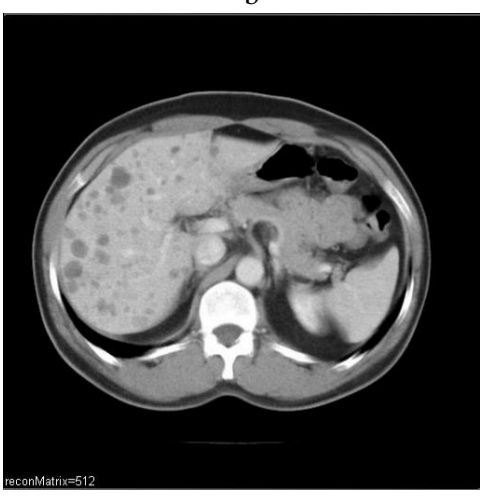

$\mathrm{e}$

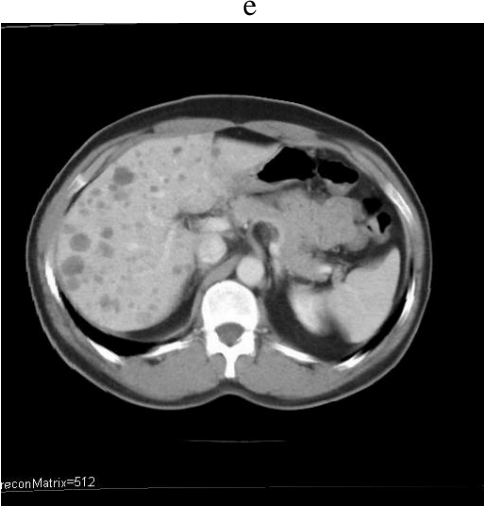

$\mathrm{h}$
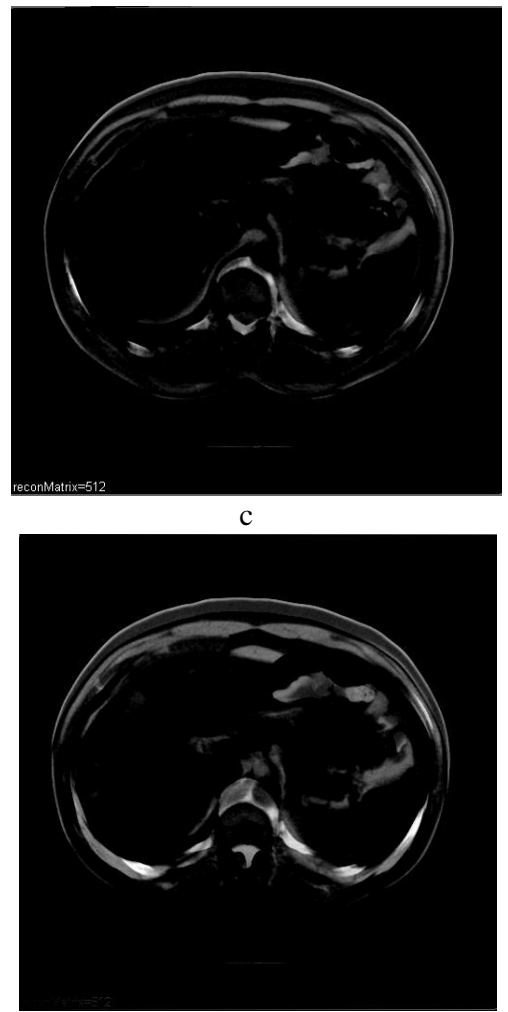

$\mathrm{f}$

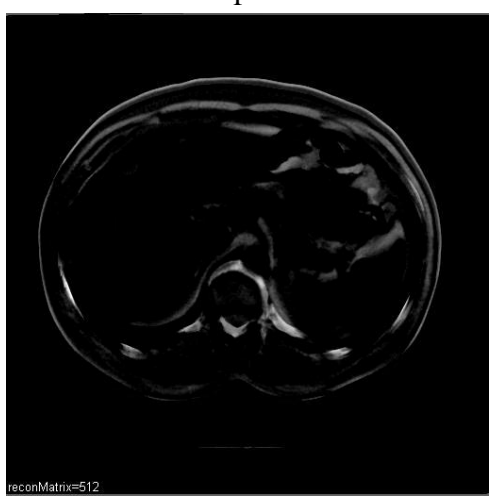

F Figure 2: Qualitative analysis for case1 with multiple metastases. (a) Pre contrast image (b)Post contrast image (c) Difference image (d) Initial registered image (e) superimposed images after initial registration (f) difference after initial registration $(g)$ hybrid registered image $(h)$ superimposed image after hybrid registration (i) 
WIPRO GE CT/e machine. Figure 2 shows results of case 1 for multiple liver metastases for a patient. Figure 2(a) and (b) are the pre and post contrast images respectively. Figure 2(c) is their difference image. The initial registered image is shown in Figure 2(d) after correcting global deformations. Figure 2(e) is initial registered image superimposed on reference image. Difference of pre contrast and initial registered image appears minimized in Figure 2(f). Figure 2(g) is the hybrid registered image and Figure 2(h) is obtained after superimposing it on Figure 2(a). Differences are further reduced in Figure 2(h) after hybrid registration.
Figure 3 shows results of case 2 with Ascitis or fluid collection in the liver of a patient. Figure 3(a) is the pre contrast image and Figure 3(b) post contrast image. Figure 3(c) is their difference image. Figure 3(d) is the initial registered image after correcting global deformations. Figure 3(e) is initial registered image superimposed on reference image. Difference is minimized in Figure 3(f). Figure 3(g) is the hybrid registered image and Figure $3(\mathrm{~h})$ is obtained after superimposing it on Figure 3(a). Differences are further reduced

in

Figure

3(h).

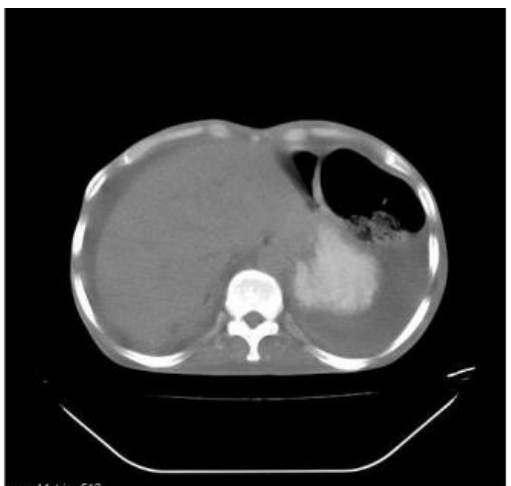

(a)

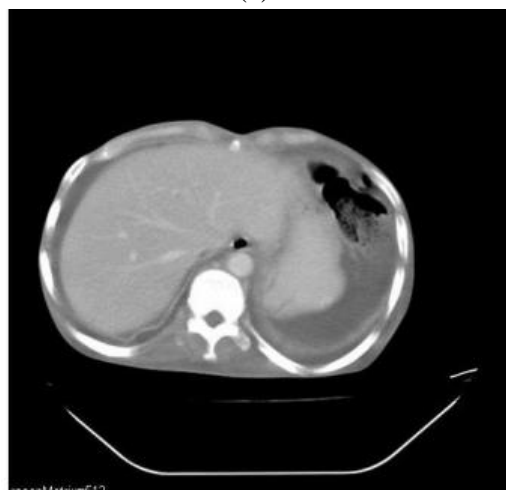

(d)

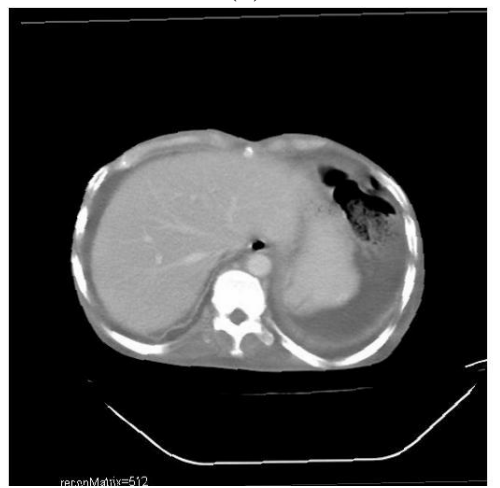

(g)

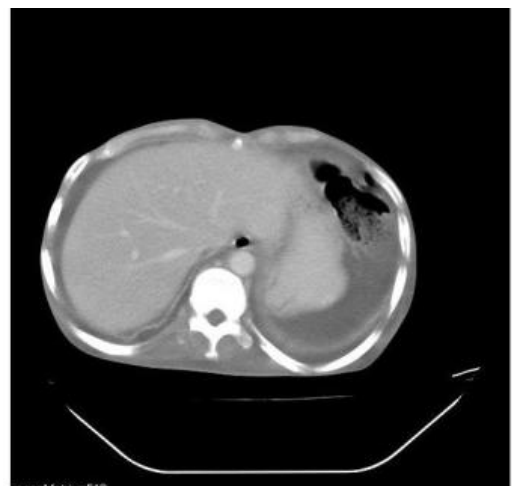

(b)

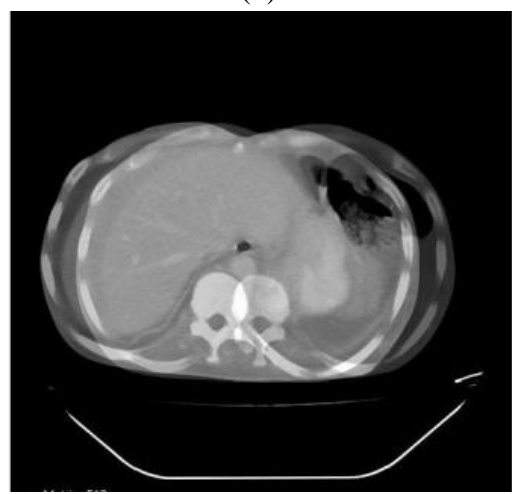

(e)

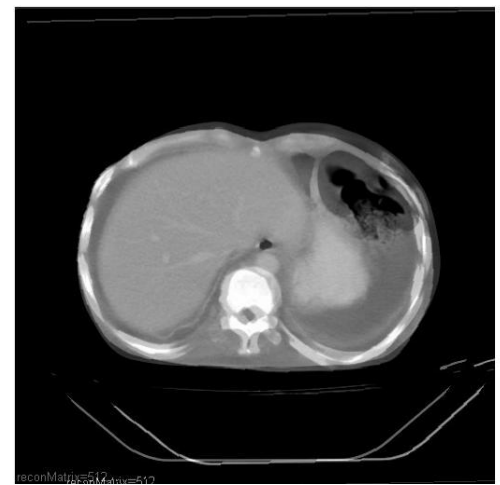

(h)

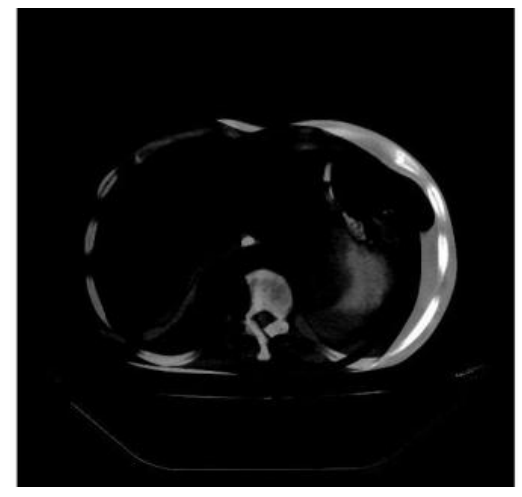

(c)

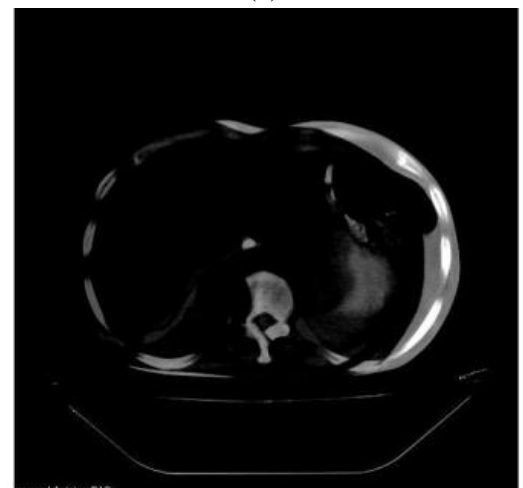

(f)

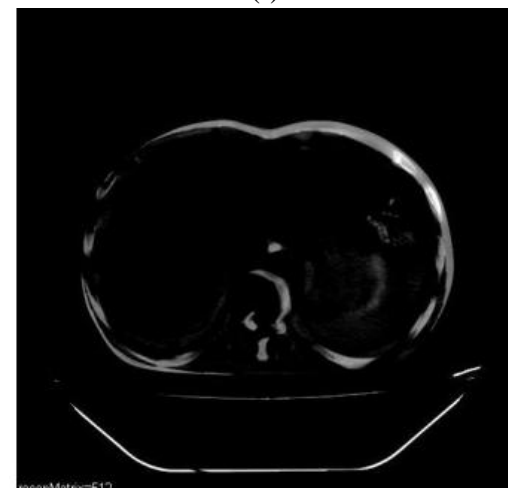

(i)

Figure 3: Qualitative analysis for case2 with Ascitis (a) Pre contrast image (b) Post contrast image (c) Difference image (d) initial registered image (e) superimposed images after initial registration (f) difference after initial registration (g) hybrid registered image (h) superimposed images after hybrid registration (i) difference after hybrid registration 
Table 1.0: Quantitative analysis for case1 and case 2

\begin{tabular}{|l|l|l|l|l|l|r|}
\hline & \multicolumn{2}{|c|}{ Correlation Coefficient } & \multicolumn{2}{c|}{ MI } & \multicolumn{2}{c|}{ MSE } \\
\hline Case & $\begin{array}{l}\text { before } \\
\text { registration }\end{array}$ & $\begin{array}{l}\text { after third level of } \\
\text { decomposition }\end{array}$ & $\begin{array}{l}\text { before } \\
\text { registration }\end{array}$ & $\begin{array}{l}\text { after hybrid } \\
\text { registration }\end{array}$ & $\begin{array}{l}\text { before } \\
\text { registration }\end{array}$ & $\begin{array}{l}\text { after hybrid } \\
\text { registration }\end{array}$ \\
\hline 1 & 0.7473 & 0.8754 & 0.8286 & 0.8455 & 33.4389 & 20.7317 \\
\hline 2 & 0.9010 & 0.9451 & 0.8057 & 0.8210 & 23.1470 & 14.7232 \\
\hline
\end{tabular}

\section{CONCLUSION}

The developed hybrid registration technique combines intensity based initial registration and landmark based elastic registration method. The hybrid registered images obtained with developed technique show considerable enhancement of lesion with prominent and well defined boundaries. Comparing successive slices in similar manner will help in better assessment of the intrusion of lesion into neighbouring anatomy. The performance of experimental work is found to be stable with variation in type of lesion, size of lesion and location of lesion within the abdomen. Variation in findings will be seen depending on size of lesion or amount of fluid collection. Increase in Correlation coefficient and MI after registration and decrease in MSE show that images are correctly registered as seen in Table 1. Qualitative analysis shows that differences are much reduced after hybrid registration. Improvement in spatial alignment is obvious from superimposition of registered image on pre contrast image.

\section{ACKNOWLEDGEMENTS}

We sincerely acknowledge the efforts of Dr. P.K Dave, MD, DRM, Radiologist, Kamla Nehru Gas Relief Hospital Bhopal, India, for his support and authorities of Jawaharlal Nehru Cancer Hospital and Research Center Bhopal, India, for providing the images for the case study.

\section{REFERENCES}

[1] L.G Brown, "Image Registration Techniques", ACM Computing Surveys, 1992, vol. 24, No.4, pp. 327-375

[2] W.R. Crum, T. Hartkens, D.L.G Hill, Non-rigid Image Registration: theory and Practice, The British Journal of Radiology, 77, pp. S140-S153, 2004.

[3] Derek L.G. Hill, Philipp G. Batchelor, Mark. Holden, and,David J. Hawkes, "Medical Image Registration", Physics in Medicine and Biology, Institute of Physics, Publishing, Vol.46, pp. R1-R45, 2001.

[4] Sharma, K.K and Joshi, S.D December 2006. Image Registration Using Fractional Fourier Transform. In proceedings of IEEE Asia Pacific Conference on Circuits \& Systems, pp. 470-473, 4-7

[5] Josien P.W. Pluim, J. Michael Fitzpatrick "Image Registration", IEEE Transactions on Medical Imaging, November 2003, Vol. 22, No.11, pp.1341-1343.

[6] T. Makela, P. Clarysse, O. Sipila, N. Pauna , Quoc Cuong Pham, T. Katila, I. E Magnin, "A Review of Cardiac Image Registration Methods", Cardiac
Imaging, IEEE Transactions, vol. 21, 9, pp.1011-1021, 2002.

[7] Langlotz, F. 2006 Computer Assissted Minimally Invasive Spine Surgery. A surgical manual, xx, 496p

[8] Kurnaz, M. N., Dokur Z. and Olmez, T. 2003. Segmentation of Ultrasound Images by Using Wavelet Transform. In proceedings of the 25th IEEE Annual International Conference of the EMBS, Cancum, Mexico, pp.657-659, 17th - 21st

[9] Kenji Suzuki, Hiroyuki Abe, Heber MacMahon, and Kunio Doi, "Image-Processing Technique for Suppressing Ribs in Chest Radiographs by Means of Massive Training Artificial Neural Network (MTANN)", IEEE Transactions on Medical Imaging, April 2006, Vol. 25, No. 4, pp. 406-416

[10] B. Ma, R. E. Ellis, "Robust Registration for Computerintegrated Orthopedic Surgery: Laboratory Validation and Clinical Experience", Medical Image Analysis, 2003, Vol. 7, pp.237-250

[11] Erik H. W. Meijering, Karel J. Zuiderveld, Wiro J. Niessen, and Viergever Max A. 1999. A fast Image Registration Technique For Motion Artifact Reduction in DSA. In proceedings of IEEE International Conference on Image Processing - IEEE Computer Society Press, Los Alamitos, CA, vol. III, pp. 435-439

[12] Pezhman Foroughi and Purang Abolmaesumi. Elastic registration of 3D Ultrasound Images 2005. In proceedings of $8^{\text {th }}$ International conference, Palm springs, CA, USA, Medical Image computing and Computer assisted intervention MICCAI October , LNCS, vol. 3749, pp. 83-90

[13] Pezhman Foroughi, Purang Abolmaesumi, Keyvan Hashtrudi-Zaad, "Intra Subject Elastic Registration of 3D ultrasound images", Medical Image Analysis, 2006, Vol.10, pp. $713-725$.

[14] D. Ruckert, L. I. Sonoda, C.Hayes, D.L.G Hill, M.O Leach and D.J Hawkes, "Nonrigid Registration Using Free Form Deformations: Application to Breast MR Images", IEEE transactions on Medical Imaging, August 1999. Vol.18, No.8, pp. 712-722

[15] Bin Fang, Yuan Yan Tang, "Elastic Registration of Retinal Images Based on Reconstructed vascular Trees", Communications, IEEE Transactions on Biomedical Engineering, , June 2006, Vol. 53, No.6, pp. 1183-1187

[16] J. B. Antoine Maintz and Max A. Viergever, "A Survey of Medical Image Registration", Medical Image Analysis, 1998, Vol.2, No.1, pp.1-37

[17] Wang, Xiu Ying., Feng, David Dagan., and Jin Jesse. 2001. Elastic Medical Image Registration Based on Intensity. In proceedings of Pan Sydney Area Workshop 
on Visual Information and Processing, Sydney, Vol.11, pp. $\quad 139-142$

[18] Wang, Xiuying and Feng, David Dagan. 2004, In proceedings of International Symposium on Intelligent multimedia, Video and Speech Processing, Hong Kong, pp.731-734, 20-22

[19] B. Likar and F. Pernus, "A Hierarchical approach to elastic registration based on mutual information", Image and Vision Computing, 2001.Vol.19, pp.32- 45

[20] Xu, Xiaoyan. and Dony, Robert D. 2004. Evaluation of Hierarchical Elastic Medical Image Registration Method. In proceedings of Canadian Conference On Electrical and Computer Engineering, vol.3 (2-3), pp.1289 -1292

[21] Jingfeng Han, Joachim Hornegger, Torsten Kuwert,Werner Bautz Wolfgang Romer, "Feature Constrained Non-rigid Image Registration”, pp.1-6, 2005.

[22] Wang, Xiuying. and Feng, David Dagan 2004. Automatic Hybrid Registration For 2-Dimensional CT Abdominal Images. In proceedings of The Third International Conference on Image and Graphics, pp. $208-211$

[23] Zhiyong Xie and Gerald E. Farin, "Image Registration Using Hierarchical B-Splines", IEEE on Visualization and Computer Graphics, January/February 2004,Vol. 10, No.1, pp.85-94

[24] Jan Kybic, Michael Unser, "Fast Parametric Elastic Image Registration", IEEE Transactions on Image Processing, November 2003.Vol. 12, No. 11, pp.14271442

[25] Panos Kotsas, "Non Rigid Registration of Medical Images using an Automated Method", Transactions on Engineering, Computing and Technology, ENFORMATIKA, V7, pp. 199-201, August 2005.
[26] Li, Dandan., Wang, Yan., Liu, Zhiyan and Jin, Jing. 2005. 3D Registration of Ultrasound Images Based on Morphology Skeleton. In proceedings of the 2005 IEEE Engineering in Medicine and Biology 27th Annual Conference Shanghai, China, September 1-4, pp. 6309 6312

[27] Lyubomir Zagrochev and Ardeshir Goshtaby, "A Comparative Study of transformation Functions for Nonrigid Image Registration", IEEE Transactions on Image Processing, 2006. Vol. 15, No. 3, pp. 529-538

[28] Chen, Hua Mei. And Varshney, Pramod K. 2000. A Pyramid Approach for Multi modality image Registration Based on Mutual Information. In proceedings of $3^{\text {rd }}$ International Conference on Image Fusion, Vol.1, pp. MOD 3/9-Mod 315

[29] Asmita A. Moghe, Jyoti Singhai, S.C Shrivastava, "Automatic Threshold Based Liver Lesion Segmentation in Abdominal 2D CT Images", International Journal of Image Processing, Vol.5, No.2, 2011, pp.166-176, ISSN (Online) 1985-2304.

[30] Moghe, Asmita A., Singhai, Jyoti. and Shrivastava, S.C. 2010. Elastic Registration of 2D Abdominal CT Images Using Hybrid Feature Point Selection for Liver Lesions. In proceedings of IEEE $2^{\text {nd }}$ International Advance Computing Conference", Patiala, India $19^{\text {th }}-20^{\text {th }}$, pp. 337-341

[31]Fred. L Bookstein, "Principal Warps: Thin Plate Splines in Decomposition of Deformations", IEEE Transactions on Pattern Analysis and Machine Vision 1989, Vol. 11, No.6, pp.567-585 